\title{
LESÃO CEREBRAL PENETRANTE POR GRANDE FRAGMENTO DE FIBRA DE AMIANTO TRATADA POR CRANIECTOMIA DESCOMPRESSIVA
}

\author{
Relato de caso
}

\author{
Gustavo Cardoso de Andradeㄹ, Roberto Leal Silveira², Aluízio Augusto Arantes J r ${ }^{3}$, \\ Gilberto Almeida Fonseca Filho ${ }^{3}$, Nilson Pinheiro J $r^{3}$
}

\begin{abstract}
RESUMO - Relata-se caso de paciente de 22 anos vítima de traumatismo cranioencefálico penetrante por fragmento de fibra de amianto medindo $15 \times 12 \mathrm{~cm}$, e seu tratamento bem sucedido por craniectomia descompressiva. Ao contrário da lesão encefálica por projétil de arma de fogo, lesão encefálica penetrante por objeto de baixa energia é incomum. A maioria dos casos relatados na literatura envolve lesões cranioorbitárias ou autoflagelação em pacientes psiquiátricos. O caso relatado torna-se especial em virtude das grandes dimensões do objeto penetrante, do tratamento por craniectomia descompressiva e do bom resultado funcional alcançado.
\end{abstract}

PALAVRAS-CHAVE: lesão cerebral penetrante, craniectomia descompressiva, corpo estranho, traumatismo cranioencefálico.

\begin{abstract}
Penetrating brain injury due to a large asbestos fragment treated by decompressive craniectomy: case report

ABSTRACT - We report the case of a 22-year-old man victim of penetrating brain injury due to a $15 \times 12$ asbestos fragment and a successfully treatment via decompressive craniectomy. Unlike gunshot wounds to the head, penetrating brain injury from low energy objectsare unusual. Most casesreported involve cranioorbitary injuries as well as self inflicted lesions in mentally ill patients. The reported case is noteworthy due to the large dimensions of the foreign body, the treatment via decompressive craniectomy and the good patient functional outcome.
\end{abstract}

KEY WORDS: penetrating brain injury, decompressive craniectomy, foreign body, head injury.

A lesão cerebral penetrante por objetos de baixa energia cinética é incomum na prática neurocirúrgica. Diversos objetos foram relatados na literatura como causadores de lesão encefálica penetrante, sendo habitualmente de pequenas dimensões e com entrada pela face. Desde o caso de Phineas Gage, ocorrido em 1848, o relato dessas lesões sempre desperta interesse.

Relatamos o caso de homem jovem vítima de traumatismo cranioencefálico por corpo estranho de baixa energia e grandes dimensões, seu tratamento bem sucedido por craniectomia descompressiva e o bom resultado funcional atingido.

\section{CASO}

Homem de 22 anos de idade, artesão, admitido no Pronto Socorro do Hospital João XXIII vítima de acidente de trabalho com traumatismo cranioencefálico penetrante por fragmento de fibra de amianto ("disco de polir pedra sabão"). À admissão, encontrava-se em estado de choque (pressão arterial sistólica de $60 \mathrm{mmHg}, 26$ incursões respiratórias por minuto), em respiração espontânea e Glasgow 8, sem déficit neurológico focal. Apresentava corpo estranho penetrante no crânio com lesão temporofronto-orbitária direita, com herniação de tecido encefálico e líquor pelas bordas da lesão (Fig 1). Após intubação orotraqueal sob sedação e reposição volêmica, foi encaminhado à tomografia computadorizada do encé-

NEUROCENTER - Serviço de Neurocirurgia do Hospital Madre Teresa / BH. Pronto Socorro do Hospital J oão XXIII / BH, Belo Horizonte MG, Brasil: ${ }^{1}$ Residente em Neurocirurgia do Hospital Madre Teresa, Plantonista do Hospital João XXIII; ${ }^{2}$ Chefe do serviço de Neurocirurgia do Hospital Madre Teresa; ${ }^{3}$ Neurocirurgião do Hospital Madre Teresa.

Recebido 5 Janeiro 2004, recebido na forma final 13 Maio 2004. Aceito 10 Julho 2004.

Dr. Gustavo Cardoso de Andrade - Rua Cachoeira de Minas 114/701 - 30440-450 Belo Horizonte MG - Brasil. E-mail: gca@zipmail.com 


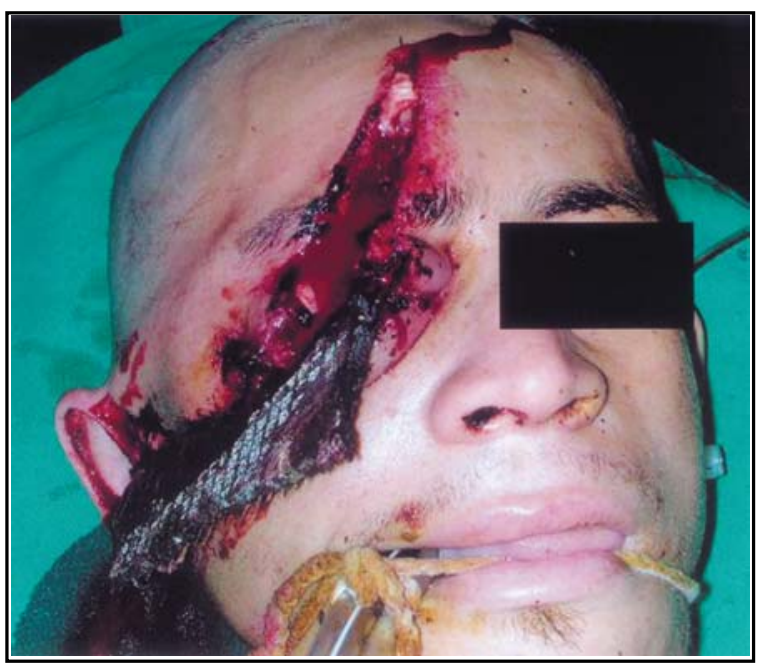

Fig 1. Lesão cerebral penetrante fronto-temporo-orbital em paciente de 22 anos.

falo (TC), que revelou extenso corpo estranho temporofronto-orbitário, com importante edema perilesional, as sociado a fraturas orbitárias, de processo mastóide e arco zigomático (Fig 2).

O tratamento neurocirúrgico consistiu em extensão fronto-parietal da ferida cutânea, ampla craniectomia temporo-fronto-parietal e abertura dural expondo todo o tecido cerebral em torno do corpo estranho antes de sua retirada (Fig 3). Retirou-se o fragmento medindo 15 x 12 centímetros sem sangramento significativo e sem intercorrências (Fig 4). Apóssua exérese procedeu-se des bridamento criterioso com retirada de partículasósseas, fragmentos do corpo estranho, aspiração de tecido desvitalizado e fechamento dural expansivo com interpo- sição de pericrânio autólogo. As células aéreas da mastóide foram tamponadas com músculo temporal pediculado e cola biológica. A síntese cutânea foi realizada por planos. O "flap" ósseo foi inserido no tecido subcutâneo abdominal no flanco direito. Ao final do procedimento cirúrgico, instalou-se dispositivo de monitorização da pressão intracraniana (parafuso de Richmond) na região frontal esquerda.

Encaminhado ao Centro de Tratamento Intensivo (CTI), onde apresentou instabilidade hemodinâmica no pós-operatório imediato, sendo tratado com aminas vasoativas cuja dosagem foi diminuída progressivamente durante três dias, visando a manutenção de pressão de perfusão cerebral (PPC) adequada. Foi extubado no quinto dia pós-operatório, quando também foi interrompida a monitorização da PIC. Apresentou sinais de meningite bacteriana (febre, rigidez nucal, leucocitose) apóso sétimo dia, recebendo antibioticoterapia de largo espectro por 28 dias. Recebeu alta do CTI no décimo dia pós-operatório. Durante o período de monitorização da PIC, esta não excedeu $24 \mathrm{mmHg}$. Foram realizadas TC seriadas de controle, evidenciando uma área de intenso edema / tumefação local e herniação encefálica transcraniana pela área da craniectomia. Contudo, as cisternas de base sempre se mantiveram patentes e não houve desvio da linha média, indicando a ausência de herniação cerebral interna (Fig 5).

No vigésimo nono dia pós-operatório, ainda em vigência de antibioticoterapia, porém sem indíciosclínicos ou laboratoriais de infecção, foi submetido a cranioplastia. O intento inicial era utilizar o fragmento ósseo inserido no abdome, porém durante a exploração cirúrgica constatou-se a presença de secreção purulenta ao seu redor, optando-se por sua retirada e uso de cimento ortopédico (metil-metacrilato).

O paciente recebeu alta hospitalar no trigésimo ter-

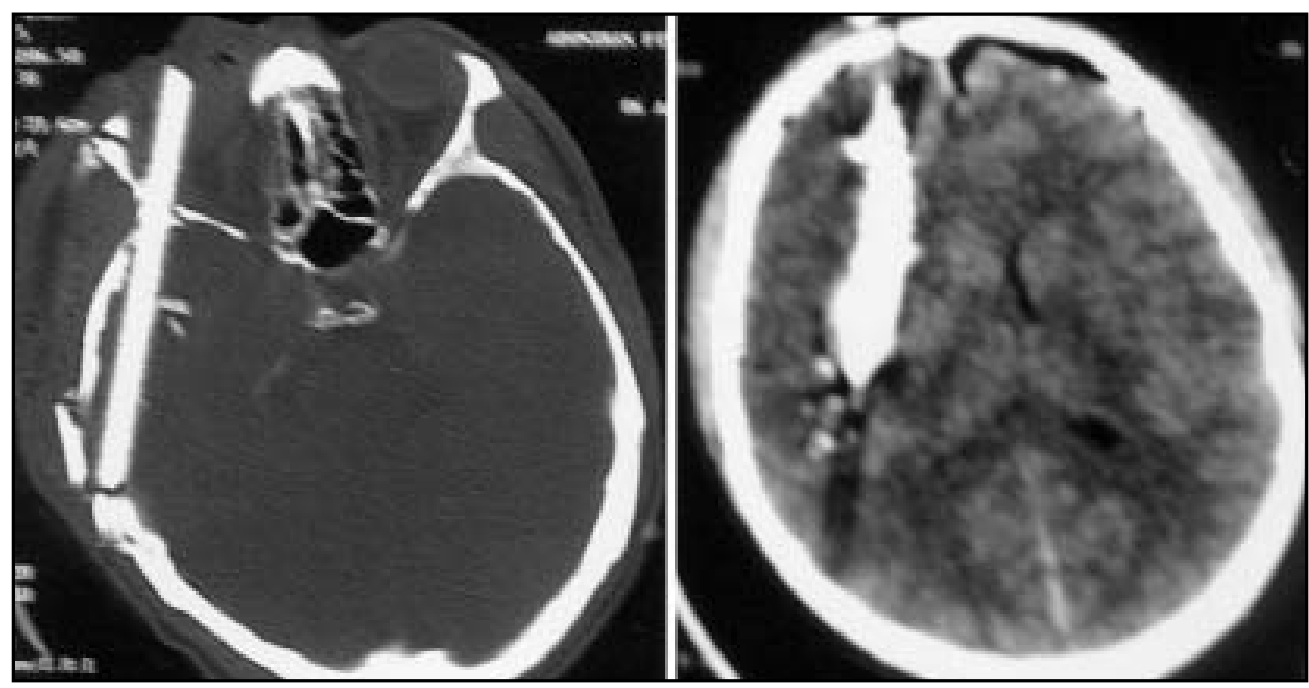

Fig 2. Tomografia computadorizada do encéfalo revelando corpo estranho temporo-fronto-orbitário, edema perilesional, associado a diversas fraturas cranianas. 


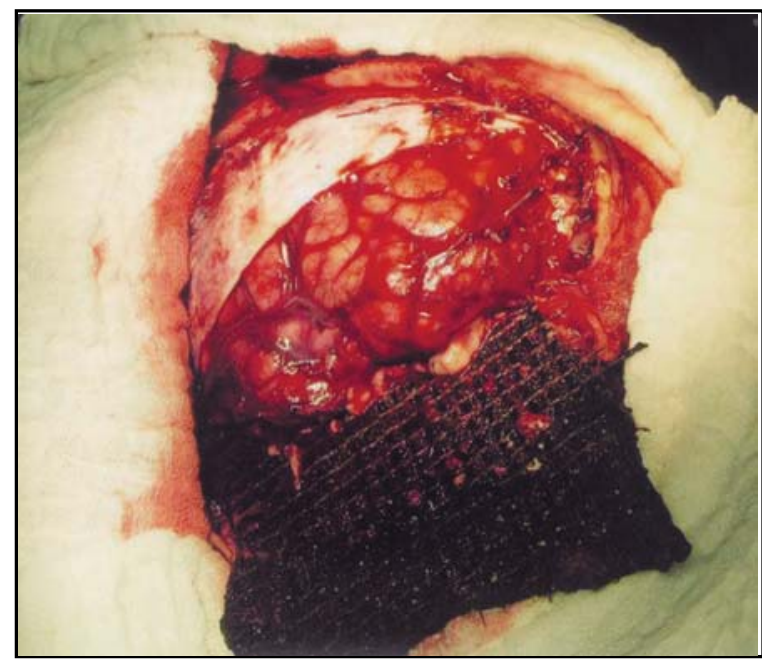

Fig 3. Craniectomia fronto-temporo-pariental expondo todo o tecido encefálico ao redor do corpo estranho.

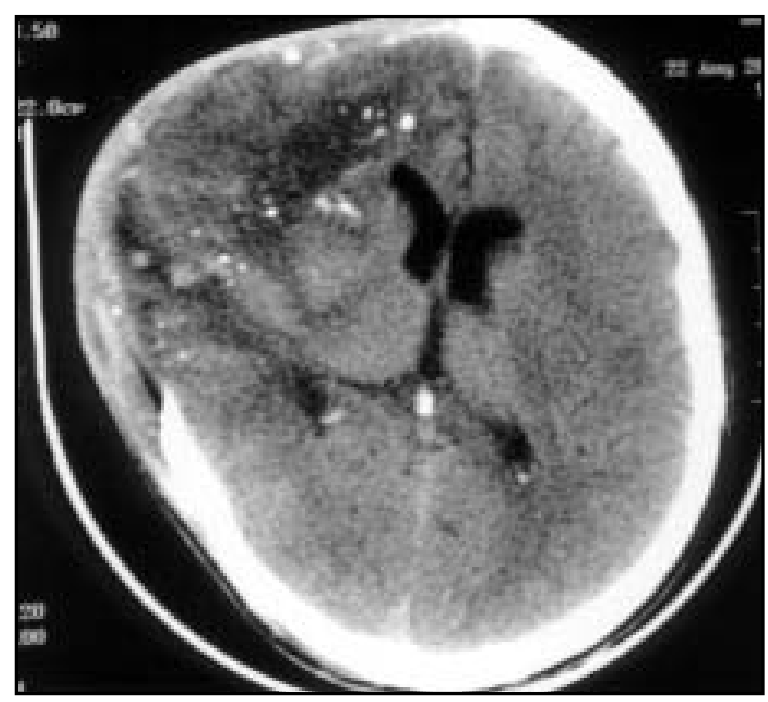

Fig 5. Tomografia computadorizada do encéfalo no pós-operatório mediato demostrando herniação transcraniana, ventrículosnormoposicionados, sem efeito de massa ou desvio de linha média.

ceiro dia após o primeiro procedimento cirúrgico. Apresentava ao exame neurológico apenas monoparesia (grau III) do membro superior esquerdo, com predomínio distal, além de amaurose e anacusia à direita, por lesão do globo ocular e ouvido médio, respectivamente. Não apresentava déficits cognitivos aparentes. Retornou para controle ambulatorial trinta diasapós alta hospitalar quando apresentou score de 23 pontos no Mini-mental, score 4 na Escala de Resultados de Glasgow e melhora progressiva da monoparesia do membro superior esquerdo (grau 4) (Fig 6).

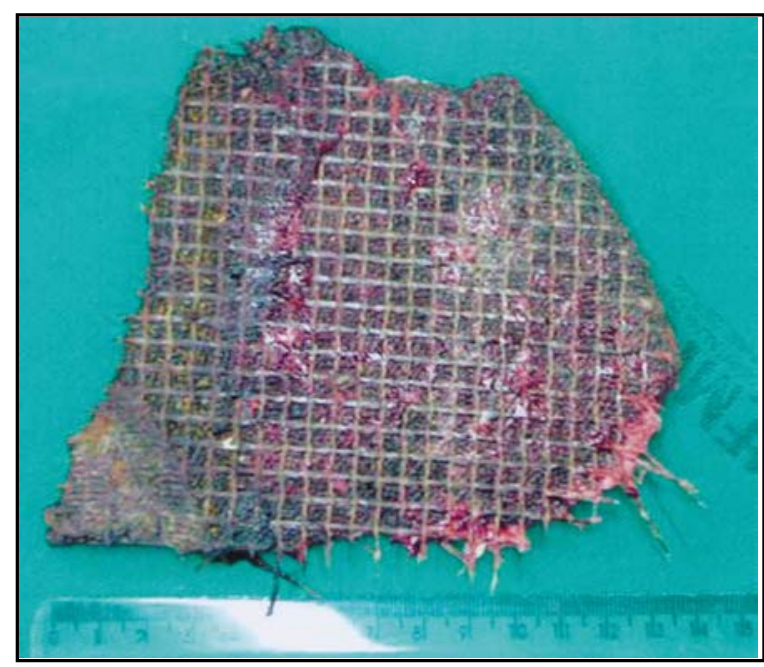

Fig 4. Fragmento de fibra de amianto (corpo estranho) medindo $15 \times 12$ cm aós sua retirada.



Fig 6. Peciente 60 dias após tratamento cirurgico. Boa recuperação funcional, apesar de monoparesia grau 4 em membro superior esquerdo.

\section{DISCUSSÃO}

A lesão cerebral penetrante de alta energia, causada por projétil de arma de fogo tem atualmente incidência crescente em todos os segmentos da sociedade civil, correspondendo, infelizmente, a evento prevalente na prática neurocirúrgica nos grandes centros urbanos ${ }^{1}$.

Por sua vez, traumatismo encefálico penetrante por objetos de baixa energia (corpos estranhos) é incomum ${ }^{1-4}$. A maioria dos casos relatados na literatura refere-se a trauma crânio-orbitário ou 
acometendo indivíduos com transtornos psiquiátricos, usualmente tratando-se de objetos pequenos $^{5-19}$. Sua ocorrência, seu tratamento e conseqüências despertam interesse desde 1848 quando Phineas Gage sobreviveu a um trauma penetrante por barra de ferro atingindo seu lobo frontal esquerdo, tornando-se um marco no estudo da função do lobo frontal e da localização cerebral ("brain mapping" ${ }^{4,20-22}$. O caso aqui relatado torna-se especial em virtude das grandes dimensões do objeto penetrante $(15 \times 12 \mathrm{~cm})$ e do bom resultado funcional alcançado.

Acreditamosque a pronta indicação de craniectomia descompressiva após evidências tomográficas de tumefação cerebral e conseqüente hipertensão intracraniana possibilitou a manutenção da PIC em valores fisiológicos, favorecendo a perfusão cerebral e prevenindo injúria secundária ${ }^{23-29}$. Outros sim, a permanência em unidade de tratamento intensivo no período pós-operatório otimizou as medidas citadas, como controle da PIC e manutenção da PPC, além de permitir o tratamento adequado e em tempo hábil de complicações como instabilidade hemodinâmica e meningite ${ }^{30-33}$.

Em conclusão, apesar da extensa lesão cerebral causada por corpo estranho de grandes dimensões, o tratamento por craniectomia descompressiva associado a pós-operatório bem conduzido em unidade de tratamento intensivo possibilitou ao paciente uma boa recuperação funcional.

Agradecimento - Os autores manifestam seu reconhecimento ao cirurgião plástico Dr. Nicodemus de Arimathéa e Silva Jr. e à equipe de terapia intensiva do Hospital J oão XXIII pela dedicação na condução do caso descrito.

\section{REFERÊNCIAS}

1. Harrington $T$, Apostolides P. Penetrating brain injury. In Cooper PR, Golfinos JG (eds). Head injury, $4^{\text {th }}$ Edition. New York:McGraw-Hill, 2000:349-360.

2. Tancioni F, Gaetani P, Pugliese R, Rodriguez Y, Baena R. Intracranial nail: a case report. J Neurosurg Sci 1994;38:239-243.

3. Blank-Reid C, Reid PC. Penetrating trauma to the head. Crit Care Nurs Clin N Am 2000;12:477-487.

4. Stone JL. Transcranial brain injuries caused by metal rods and pipes over the past 150 years. J Hist Neurosci 1999;8:227-234.

5. Yamashita M, Abrahão N Jr, Lamachia C. Tentativa de suicídio pela introdução de dois pregos na cabeça: relato de caso. Arq Neuropsiquiatr 1998;56:317-319.

6. Kahler RJ, Tomlinson FH, Eisen DP, Masel JP. Orbitocranial penetration by a fern: case report. Neurosurgery 1998;42:1370-1373.
7. Domenicucci M, Qasho R, Ciapetta P, Vangelista T, Delfini R. Surgical treatment of penetrating orbito-cranial injuries: case report. J Neurosurg Sci 1999;43:229-234.

8. Sollmann WP, Seifert V, Haubitz B, Dietz H. Combined orbito-frontal injuries. Neurosurg Rev 1989;12:115-121.

9. Takanashi $Y$, Shinonaga M, Manaka H. Penetrating brain injury with nasal entry by a plastic stick: case report. J Neurosurg Sci 2002;46:25-27.

10. Matsumoto S, Hasuo K, Mizushima A, et al. Intracranial penetrating injuries via the optic canal. Am J Neuroradiol 1998;19:1163-1165.

11. Gonçalves da Silva CE, Gonçalves da Silva JA. A rare orbito-cerebral penetrating knife-wound. Neurochirurgia (Stuttg) 1979;22:28-30.

12. Lunetta P, Ohberg A, Sajantila A. Suicide by intracerebellar ballpoint pen. Am J Forensic Med Pathol 2002;23:334-337.

13. Doron $Y$, Gruszkiewicz J, Peyser E. Penetrating cranio-cerebral injuries due to unusual foreign bodies. Neurosurg Rev 1982;5:35-40

14. Leal MB Filho, Almeida BR, Aguiar ADE, Vieira MA, Morais RK, Dantas KS. Corpo estranho no cone orbitário: relato de caso. Arq Neuropsiquiatr 2003;61:490-493.

15. Stephanov S. Severe craniocerebral injury by an axe with good outcome: case report. Swiss Surg 1999;5:80-82.

16. Ohaegbulam SC, Ojukwu JO. Unusual craniocerebral injuries from nailing. Surg Neurol 1980;14:393-395.

17. Bursick DM, Selker RG. Intracranial pencil injuries. Surg Neurol 1981;16:427-431.

18. Musa BS, Simpson BA, Hatfield RH. Recurrent self-inflicted craniocerebral injury: case report and review of the literature. Br J Neurosurg 1997;11:564-569.

19. Barros RS, Silveira RL, Cabral G Filho. Fragmento de madeira intracraniano. Arq Bras Neurocir 1982;1:77-80.

20. Barker FG. Phineas among the phrenologists: the American crowbar case and nineteenth-century theories of cerebral localization. J Neurosurg 1995;82:672-682.

21. MacMillan M. Phineas Gage's contribution to brain surgery. J His Neurosci 1996;5:56-77.

22. MacMillan M. Commemorating the $150^{\text {th }}$ anniversary of Phineas Gages's accident. 0J Hist Neurosci 2000;9:90-93.

23. Schneider GH, Bardt T, Lanksch WR, Unterberg A. Decompressive craniectomy following traumatic brain injury: ICP, CPP and neurological outcome. Acta Neurochir 2002;81(Suppl):77-79.

24. Carmelo A, Ficola A, Fravolini ML, et al. ICP and CBF regulation: effect of the deompressive craniectomy. Acta Neurochir 2002;81(Suppl):109-111.

25. Coplin WM, Cullen NK, Policherla PN, et al. Safety and feasibility of craniectomy with duraplasty as the initial surgical intervention for severe traumatic brain injury. J Trauma 2001;50:1050-1059.

26. Munch E, Horn P, Schurer L, Piepgras A, Paul T, Schmiedek P. Management of severe traumatic brain injury by decompressive craniectomy. Neurosurgery 2000;47:315-322

27. Guerra WK, Gaab MR, Dietz H, Mueller JU, Piek J, Fritsch MJ. Surgical decompression for traumatic brain swelling: indications and results. J Neurosurg 1999;80:187-196

28. Pereira WC, Neves VJ, Rodrigues Y. Craniotomia descompressiva bifrontal no tratamento de edema cerebral grave. Arq Neuropsiquiatr 1977;35:99-111.

29. Silva JA, Silva CE, Sousa MB. Craniotomia descompressiva no edema cerebral grave: a propósito de 30 casos operados. Arq Neuropsiquiatr 1976;34:232-240.

30. Flannery T, McConnell RS. Cranioplasty: why throw the flap out? Br J Neurosurg 2001;15:518-520.

31. Stula D, Muller HR. Cranioplasty after extensive decompressive craniotomy with displacement of the cerebral hemisphere: CA analysis. Neurochirurgia (Stuttg) 1980;23:41-46.

32. Ziai WC, Port JD, Cowan JA, Garonzik IM, Bhardwaj A, Rigamonti D. Decompressive craniectomy for intractable cerebral edema: experience of a single center. J Neurosurg Anesthesiol 2003;15:25-32.

33. Piek J. Decompressive surgery in the treatment of traumatic brain injury. Curr Opin Crit Care 2002;8:134-138. 